\title{
Efeito do treinamento sobre a cinemática de equinos no salto de obstáculos
}

\author{
[Effects of training on kinematic traits of jumping horses] \\ E. Schlup ${ }^{1,2}$, F.N. Godoi ${ }^{1}$, A.M. Andrade ${ }^{1}$, J.A.G. Bergmann ${ }^{3}$, F.Q. Almeida ${ }^{1 *}$ \\ ${ }^{1}$ Universidade Federal Rural do Rio de Janeiro - UFRRJ - Seropédica, RJ \\ ${ }^{2}$ Escola de Equitação do Exército - EsEqEx - Rio de Janeiro, RJ \\ ${ }^{3}$ Universidade Federal de Minas Gerais - Escola de Veterinária - UFMG - Belo Horizonte, MG
}

\begin{abstract}
RESUMO
Este trabalho teve como objetivo avaliar o efeito do treinamento sobre as variáveis da cinemática de equinos jovens da raça Brasileiro de Hipismo no salto em liberdade. Foram utilizados 14 equinos da raça Brasileiro de Hipismo com idade de 40 a 42 meses, peso vivo de 436,3 $\pm 11,6 \mathrm{~kg}$ e 448,2 $\pm 12,7 \mathrm{~kg}$ e altura na cernelha de $1,57 \pm 0,03 \mathrm{~m}$ e $1,58 \pm 0,03 \mathrm{~m}$ ao início e ao final do experimento, respectivamente. Os equinos realizaram dois protocolos de avaliação de salto, um antes e outro após o treinamento. O treinamento teve a duração de cinco meses e consistiu em 50 minutos de atividade física por dia, montados, sendo dois dias de flexionamento, dois dias de condicionamento físico e dois dias de trabalho específico de salto de obstáculos. Nos protocolos de avaliação, foram filmados cinco saltos em liberdade sobre um obstáculo oxer, com o primeiro e o segundo elemento na altura de $0,90 \mathrm{~m}$ e $1,00 \mathrm{~m}$ de altura, respectivamente, e $0,90 \mathrm{~m}$ de largura. Foram fixados marcadores reflexivos nos equinos em pontos anatômicos utilizados como pontos de referência para a avaliação das características cinemáticas no salto. As filmagens foram realizadas com câmera de $100 \mathrm{~Hz}$, e as imagens, processadas no Simi Reality Motion Systems $^{\circledR}$. Os resultados das variáveis avaliadas foram comparados com o teste de $t$ pareado $(\mathrm{P}<0,05)$. $\mathrm{O}$ treinamento resultou no aumento das variáveis $(\mathrm{P}<0,05)$ : velocidade da passada anterior ao salto, velocidade do salto, deslocamento horizontal da cernelha no salto, ângulo escapuloumeral, distância vertical entre as articulações escapuloumeral e metacarpofalângica, ângulo coxofemoral e ângulo femorotibial. No entanto, os valores dos ângulos umerorradial e tibiometatarsiano apresentaram redução após o treinamento $(\mathrm{P}>0,05)$. Conclui-se que o treinamento específico para salto com cinco meses de duração altera algumas variáveis cinemáticas dos equinos no salto de obstáculo proporcionando melhoria, principalmente, na trajetória do salto.
\end{abstract}

Palavras-chave: cavalo, biomecânica, hipismo

\begin{abstract}
This study was carried out to evaluate the effect of training on the kinematic traits of young Brazilian Sport Horse in free jumping. 14 Brazilian Sport Horse were used, aged between 40 and 42 months, with body weight of $436.3 \pm 11.6 \mathrm{~kg}$ and $448.2 \pm 12.7 \mathrm{~kg}$ and height withers of $1.58 \pm 0.03 \mathrm{~m}$ and $1.58 \pm 0.03 \mathrm{~m}$ at the beginning and end of training, respectively. Two jumping evaluation protocols were used: before and after training. The training had the duration of five months and consisted of 50 minutes of exercise activities by day with a rider; two days of flexing, two days of physical conditioning and two days of specific jumping exercises. The evaluations were carried out, and five free jumps in each evaluation were record in an oxer, with the front fence of $0.90 \mathrm{~m}$ and the back fence of $1.00 \mathrm{~m}$ height and $0.90 \mathrm{~m}$ of width. The horse's anatomical points were highlighted by reflective markers. The recordings were performed with $100 \mathrm{~Hz}$ camera and the images processed in Simi Reality Motion Systems ${ }^{\circledR}$. The results were analyzed as paired data $(P<0.05)$. The training increased the traits kinematics $(P<0.05)$ : last stride velocity speed prior to jumping, jumping velocity, horizontal withers displacement at jumping, scapulohumeral angle,
\end{abstract}

Recebido em 4 de março de 2014

Aceito em 12 de novembro de 2014

*Autor para correspondência (corresponding author)

E-mail: almeidafq@yahoo.com.br 


\section{Schlup et al.}

vertical distance between scapulohumeral and metacarpophalangeal joint, coxofemoral angle and femorotibial angle. However, the kinematic traits reduced with training: humeroradial angle and tibiometatarsal angle $(\mathrm{P}<0.05)$. In conclusion, the jumping training with a five month duration changed some kinematic traits of free jumping in horses, especially the jumping trajectory.

Keywords: equine, biomechanics, show-jumping

\section{INTRODUÇÃO}

No hipismo, observa-se crescente investimento na seleção e pré-seleção de equinos visando à otimização da gestão de recursos com aporte financeiro em animais com maior propensão a terem resultados positivos (Koenen et al., 2004). Porém, a escolha cada vez mais precoce dos equinos induz a questões referentes à influência do treinamento nos resultados futuros dos animais: seria a habilidade em desportos equestres inata ou esta pode ser desenvolvida somente com o treinamento? Ou até que ponto o treinamento pode corrigir eventuais imperfeições dos equinos jovens? (Santamaría et al., 2006).

A dificuldade de realizar um treinamento padronizado, bem como a utilização de equinos com características semelhantes, é um entrave nas pesquisas com treinamento de equinos (Santamaría et al., 2004). Outro fator pertinente é a duração do treinamento, pois os equinos estão em contínua fase de aprendizado, não sendo possível estimar a duração de um programa de treinamento, além de outros fatores, como lesões e condicionamento físico, interferirem no rendimento do treinamento.

Os protocolos de avaliação do salto dos equinos são utilizados, principalmente na seleção de garanhões, com os animais saltando em liberdade, ou seja, sem o cavaleiro, de modo a diminuir a intervenção do homem na biomecânica natural do equino (Lewczuk, 2008). Em termos experimentais, algumas diferenças observadas nos resultados das pesquisas podem ser associadas à qualidade do equino no salto ou às diferentes alturas dos obstáculos, em diferentes tipos de esforços (submáximo ou esforço diário) e, principalmente, aos treinamentos dos animais (Wejer et al., 2013). No presente estudo, objetivou-se avaliar o efeito do treinamento sobre as variáveis da cinemática de equinos jovens da raça Brasileiro de Hipismo no salto em liberdade.

\section{MATERIAL E MÉTODOS}

Foram utilizados 14 equinos da raça Brasileiro de Hipismo da Escola de Equitação do Exército, Rio de Janeiro, com idade de 40 a 42 meses. Os animais apresentavam no início do estudo o peso vivo de $436,3 \pm 11,6 \mathrm{~kg}$ e altura na cernelha de $1,57 \pm 0,03 \mathrm{~m}$ e, ao término do estudo, o peso vivo de $448,2 \pm 12,7 \mathrm{~kg}$ e altura na cernelha de $1,58 \pm 0,03 \mathrm{~m}$. Os animais possuíam doma e pouca experiência no salto de obstáculo.

Os equinos realizaram dois protocolos de avaliação de salto, um antes e outro após cinco meses de treinamento. Os protocolos foram realizados de forma similar, em pista coberta de 30x70m, com piso de areia. Foram afixados 19 marcadores reflexivos, no lado esquerdo dos animais (Fig. 1), que foram utilizados como pontos de referência para a análise cinemática dos equinos durante o salto.

O obstáculo utilizado nas análises foi o Oxer, que é constituído de dois elementos dispostos em ordem crescente, $0,90 \times 1,00 \mathrm{~m}$ de altura e $0,90 \mathrm{~m}$ de largura, para serem transpostos pelos equinos em um único salto (Confederação..., 2014). Esse obstáculo foi escolhido por exigir que os equinos realizem o salto tanto em altura como em largura. Visando facilitar o salto dos equinos inexperientes, foi montado um obstáculo, como referência, em forma de “ $\mathrm{X}$ ”, distando 6,00m do obstáculo Oxer.

Foi utilizada uma câmera Basler A602fc, com frequência de aquisição de imagens de $100 \mathrm{~Hz}$, a $15 \mathrm{~m}$ do obstáculo oxer, conectada ao computador, e as imagens foram processadas no Simi Reality Motion Systems ${ }^{\circledR}$. No local das filmagens ainda foram instaladas quatro fontes de luz com 1.000W de potência cada, ajustadas conforme a necessidade de iluminação, e um pano preto de $15 \times 8 \mathrm{~m}$ ao fundo para proporcionar melhor nitidez às imagens. Foram filmados cinco saltos com sucesso de cada equino, ou seja, apenas os saltos que não tiveram nenhum tipo de penalidade (desvio, refugo ou derrube de obstáculo). 


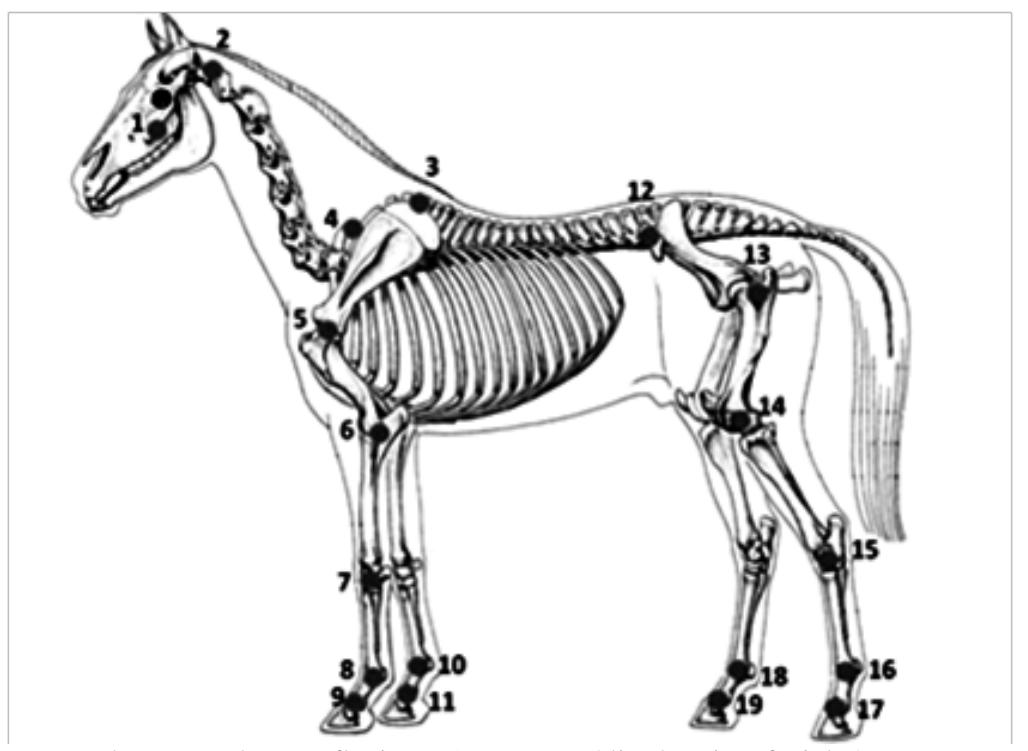

Figura 1. Posicionamento dos marcadores reflexivos: 1) ponto médio da crista facial; 2) porção cranial da face lateral da asa do atlas; 3) porção dorsal na cartilagem da escápula seguindo a linha da espinha da escápula; 4) ponto médio da borda cranial da escápula, cranialmente ao músculo supra-espinhoso; 5) área central da articulação escapuloumeral, na região da cavidade glenoide da escápula e cabeça do úmero; 6) área central da articulação umerorradial, na região do côndilo do úmero, fóvea capitular do rádio e incisura troclear da ulna; 7) terço médio lateral da articulação cárpica, região lateral do osso carpiano ulnar; 8) terço médio da face lateral da articulação metacarpofalângica do membro torácico esquerdo; 9) face lateral da articulação interfalângica proximal do membro torácico esquerdo; 10) terço médio da face medial da articulação metacarpofalângica do membro torácico direito; 11) face medial da articulação interfalângica proximal do membro torácico direito; 12) ponto médio ventral da face lateral da tuberosidade coxal; 13) região média do trocanter maior do fêmur, na articulação coxofemoral; 14) ponto lateral da articulação femorotibial; 15) terço médio lateral da articulação társica, na região lateral entre a base do calcâneo e osso tálo; 16) terço médio da face lateral da articulação metatarsofalângica do membro pélvico esquerdo; 17) face lateral da articulação interfalângica proximal do membro pélvico esquerdo; 18) terço médio da face medial da articulação metatarsofalângica do membro pélvico direito; 19) face medial da articulação interfalângica proximal do membro pélvico direito; em acordo com a Nomenclatura Anatômica Veterinária Ilustrada (Schaller, 1999).

Na avaliação cinemática do salto dos equinos, foram mensuradas 19 variáveis, segundo Bobbert et al. (2005), Santamaría et al. (2006), Lewczuk e Ducro (2012) e Godoi et al. (2013), descritas a seguir (Tab. 1).

Após a realização do primeiro protocolo de avaliação de salto, os equinos foram submetidos ao treinamento de salto de 50 minutos diários, montados por cavaleiros experientes, seis dias da semana, durante cinco meses. Esse período de treinamento permite que os equinos estejam aptos a realizar competições de salto com altura de $1,00 \mathrm{~m}$. O treinamento está de acordo com o método de ensino aplicado na Escola de Equitação do Exército. O trabalho semanal consistiu em dois dias de flexionamento, dois dias de condicionamento físico e dois dias de trabalho específico de salto.
No trabalho de flexionamento foi ensinado aos equinos noções básicas de adestramento e, principalmente, as ações de rédeas elementares: $1^{\circ}$ efeito (rédea de abertura) e $3^{\circ}$ efeito (rédea contrária), por se tratarem de efeitos de rédeas em que as pernas atuam sempre visando ao movimento para frente, de modo a não diminuir a impulsão do cavalo. No trabalho de condicionamento físico, foram realizados exercícios em terreno aberto ou em pista de cross-country ao trote e galope. Ao início do trabalho, os tempos de trote eram curtos (5 minutos) e intercalados com um tempo maior de passo (10 minutos). A duração da sessão de trabalho nunca foi superior a 50 minutos. Visando aumentar a exigência física, os equinos foram submetidos a variações do terreno, com rampas ascendentes e descendentes, bem como à transposição de pequenos obstáculos naturais (valas, taludes e troncos). 


\section{Schlup et al.}

Tabela 1. Descrição das variáveis avaliadas na cinemática do salto dos equinos

\begin{tabular}{ll}
\hline $\mathrm{N}^{\circ}$ & \multicolumn{1}{c}{ Nome } \\
01 & $\begin{array}{l}\text { Amplitude da passada anterior } \\
\text { ao salto }\end{array}$ \\
02 & $\begin{array}{l}\text { Velocidade da passada } \\
\text { anterior ao salto }\end{array}$ \\
03 & $\begin{array}{l}\text { Distância da batida } \\
04\end{array}$ \\
05 & Distância da recepção \\
06 & $\begin{array}{l}\text { Amplitude do salto } \\
\text { Velocidade do salto }\end{array}$ \\
07 & $\begin{array}{l}\text { Altura dos membros torácicos } \\
\text { no salto }\end{array}$ \\
08 & $\begin{array}{l}\text { Altura dos membros pélvicos } \\
\text { Altura da cernelha no salto }\end{array}$ \\
10 & $\begin{array}{l}\text { Deslocamento horizontal da } \\
\text { cernelha no salto }\end{array}$ \\
11 & Ângulo do pescoço
\end{tabular}

12 Ângulo escapuloumeral ${ }^{* * *}$

Comprimento da passada completa do membro torácico Descrição

Marcador esquerdo anterior ao salto

9

Velocidade horizontal para percorrer a variável $n^{\circ} 01$, calculada como a distância sobre tempo

Distância entre obstáculo e casco pélvico mais próximo ao obstáculo, imediatamente antes da fase de voo (decolagem)

Distância entre o obstáculo e o casco torácico que toca primeiro o solo imediatamente após a fase de voo (aterrissagem)

Mensurado pela distância entre o contato do casco pélvico esquerdo com o solo na batida até o contato deste mesmo casco com o solo na recepção (comprimento do salto)

Velocidade horizontal da variável $\mathrm{n}^{\circ} 05$, calculada como a distância sobre tempo

Altura mínima dos membros torácicos no momento da passagem sobre o segundo elemento do obstáculo

Altura mínima dos membros pélvicos no momento da passagem sobre o segundo elemento do obstáculo

Altura máxima durante a trajetória do salto até o solo

Distância horizontal do marcador 3, no momento da altura máxima da cernelha durante a trajetória do salto

Ângulo ventral formado pela interseção da linha entre a asa do atlas e a porção dorsal na cartilagem da escápula com a linha entre esse ponto e o ponto da área central da articulação escapuloumeral

Ângulo caudal formado pela interseção da linha entre a porção dorsal na cartilagem da escápula e a área central da articulação escapuloumeral com a linha entre as áreas centrais das articulações escapuloumeral e umerorradial

13 Ângulo umerorradial ${ }^{* * *}$ Ângulo cranial formado pela interseção da linha entre a área central da articulação escapuloumeral e a área central da articulação umerorradial com a linha entre a área central da articulação umerorradial e o terço médio lateral da articulação cárpica

14 Ângulo radiometacárpico ${ }^{* * *}$

Ângulo caudal formado pela interseção da linha entre a área central da articulação umerorradial e o terço médio lateral da articulação cárpica com a linha entre o terço médio lateral da articulação cárpica e o terço médio da face lateral da articulação metacarpofalângica

15 Distância escapulometacarpofalângica ${ }^{* * *}$

Distância vertical formada entre a área central da articulação escapuloumeral e o terço médio da face lateral da articulação metacarpofalângica

16 Ângulo coxofemoral ${ }^{* * *}$

Ângulo cranial formado pela interseção da linha entre a tuberosidade coxal e a articulação coxofemoral com a linha entre a articulação coxofemoral e a articulação femorotibial

17 Ângulo femorotibial ${ }^{* * *}$

Ângulo caudal formado pela interseção da linha entre a articulação coxofemoral e a articulação femorotibial com a linha entre as articulações femorotibial e a társica

18 Ângulo tibiometatarsico ${ }^{* * *}$ Ângulo cranial formado pela interseção da linha entre as articulações femorotibial e a társica, e a linha entre as articulações társica e a metatarsofalângica

19 Ângulo cernelhametarsofalângica $^{* * *}$ Ângulocranial formado pela interseção da linha entre o marcador na porção dorsal na cartilagem da escápula e o ponto médio ventral da face lateral da tuberosidade coxal com a linha entre esse ponto e o terço médio da articulação metatarsofalângica.

17 ou 19

9 ou 11

$-$

8

16

${ }^{*}$ A descrição da numeração dos marcadores está na Figura 1. ${ }^{* *}$ O deslocamento pode ocorrer anterior ou posterior ao obstáculo, sendo padronizado em valores negativos e positivos, respectivamente. ${ }^{* * *}$ As variáveis do segmento torácico e pélvico (do número 11 ao 19) foram mensuradas no momento em que o membro torácico ou pélvico estava sobre o segundo elemento do obstáculo, utilizando como referência os marcadores 8 e 16, respectivamente. 
No trabalho específico para salto, foram realizados trabalhos em dois tipos de obstáculos: obstáculos naturais (fixos) e obstáculos de pista (móveis). Os obstáculos naturais ou fixos compreendem aqueles encontrados na natureza, semelhantes aos observados nas provas de crosscountry da modalidade Concurso Completo de Equitação, tais como: buracos, valetas, troncos, banquetas e barrancos. Esse tipo de obstáculo não é derrubado pelo contato do cavalo. Os obstáculos de pista ou móveis são aqueles encontrados em provas da modalidade Salto, que são compostos por varas de madeira e podem ser derrubados pelo contato com o cavalo. O início do trabalho baseou-se em ginásticas de salto com utilização de cavaletes de trote, com o objetivo de cadenciar o animal para o salto (Allen e Dennis, 2002). Após realizar os saltos com ritmo e cadência, os equinos eram submetidos a exercícios de saltos isolados em um obstáculo de cada vez. Em seguida foram realizados trabalhos em linhas, ou seja, mais de um obstáculo em sequência, visando adaptar o equino às condições semelhantes às competições de salto.

Os resultados das variáveis avaliadas foram comparados com o teste de t pareado, com dois tratamentos (avaliação do salto antes e após o treinamento) e 14 repetições (animais), a 5\% de probabilidade.

\section{RESULTADOS E DISCUSSÃO}

Os resultados da variável amplitude da passada anterior ao salto, bem como da amplitude do salto, não apresentaram influência do treinamento $(\mathrm{P}>0,05)$ (Tab. 2). Tal fato pode ser explicado pela utilização do obstáculo de referência antes do salto, que obrigava os animais a percorrer uma distância padronizada a fim de melhor abordar o obstáculo filmado. O obstáculo de referência foi utilizado por se tratar de equinos inexperientes e também é rotineiramente utilizado em pesquisas com equinos no salto (Powers e Harrison, 2000; Santamaría et al., 2005; Santamaría et al., 2006; Lewczuk et al., 2006; Lewczuk, 2008; Godoi et al., 2014).

No presente estudo foi observado significativo aumento da velocidade horizontal, tanto na aproximação ao obstáculo quanto durante o salto $(\mathrm{P}<0,05)$. Essas alterações podem ter sido ocasionadas pelo aumento do condicionamento físico dos equinos durante o treinamento. A alteração da velocidade, em determinado andamento, pode ser realizada devido à mudança em dois fatores principais: amplitude e frequência (Barrey, 2013). Observa-se no presente estudo que a amplitude das passadas dos animais não foi alterada com o treinamento, levando à constatação de que o treinamento aumentou a frequência de repetições das passadas do galope.

As características distância da batida e distância da recepção não foram influenciadas pelo treinamento $(\mathrm{P}>0,05)$. Como não houve alteração nas características amplitude da passada anterior ao salto e amplitude do salto, seria difícil que houvesse efeito significativo do treinamento sobre os valores das distâncias da batida e recepção.

Tabela 2. Valores médios e desvio padrão das características cinemáticas lineares e velocidades dos equinos no salto de obstáculo

\begin{tabular}{lccc}
\hline & \multicolumn{2}{c}{ Avaliação dos equinos no salto } & \multirow{2}{*}{ Paracterísticas cinemáticas } \\
\cline { 2 - 3 } & $\begin{array}{c}\text { Antes do } \\
\text { treinamento }\end{array}$ & $\begin{array}{c}\text { Após o } \\
\text { treinamento }\end{array}$ & \\
\hline Amplitude da passada anterior ao salto (m) & $3,0 \pm 0,3 \mathrm{a}$ & $3,1 \pm 0,2 \mathrm{a}$ & 0,1312 \\
Velocidade da passada anterior ao salto (m/s) & $6,4 \pm 0,7 \mathrm{~b}$ & $7,0 \pm 0,4 \mathrm{a}$ & 0,0019 \\
Amplitude do salto (m) & $4,9 \pm 0,5 \mathrm{a}$ & $5,0 \pm 0,4 \mathrm{a}$ & 0,8162 \\
Velocidade do salto (m/s) & $5,4 \pm 0,7 \mathrm{~b}$ & $6,0 \pm 0,8 \mathrm{a}$ & 0,0213 \\
Distância da batida (m) & $1,4 \pm 0,2 \mathrm{a}$ & $1,4 \pm 0,3 \mathrm{a}$ & 0,5651 \\
Distância da recepção (m) & $1,7 \pm 0,4 \mathrm{a}$ & $1,8 \pm 0,3 \mathrm{a}$ & 0,6061 \\
Altura dos membros torácicos no salto (m) & $0,2 \pm 0,0 \mathrm{a}$ & $0,2 \pm 0,1 \mathrm{a}$ & 0,0557 \\
Altura dos membros pélvicos no salto (m) & $0,3 \pm 0,1 \mathrm{a}$ & $0,3 \pm 0,1 \mathrm{a}$ & 0,8123 \\
Altura da cernelha no salto (m) & $2,1 \pm 0,1 \mathrm{a}$ & $2,1 \pm 0,0 \mathrm{a}$ & 0,3276 \\
Deslocamento horizontal da cernelha no salto (m) & $0,3 \pm 0,15 \mathrm{~b}$ & $0,41 \pm 0,14 \mathrm{a}$ & 0,0009 \\
\hline
\end{tabular}

Médias seguidas por letras diferentes nas linhas diferem pelo teste de $\mathrm{t}(\mathrm{P}<0,05)$; Prob.: probabilidade estatística. 
As alturas dos membros torácicos e pélvicos sobre o obstáculo são as variáveis que estão diretamente relacionadas com penalidades ou o derrube do obstáculo. Animais que apresentam maiores valores dessas variáveis tendem a ter menor possibilidade de cometer penalidades. No presente trabalho não foram observadas diferenças significativas na altura dos membros torácicos e pélvicos sobre o obstáculo antes e após o treinamento.

Santamaría et al. (2006) e Lewczuk (2008) observaram efeito do treinamento nas características supracitadas utilizando protocolos diferentes para cada estágio do treinamento. Há de se considerar, entretanto, que, ao modificar as alturas, e até mesmo o protocolo de execução, não se isola a variável treinamento, inserindo-se mais uma possibilidade de interferência nos resultados. A utilização de saltos com altura máxima do obstáculo simulando uma prova de potência, não foi considerada uma escolha adequada por se tratarem de equinos inexperientes e poderia causar lesões nos mesmos.

Houve efeito no deslocamento horizontal da cernelha no salto $(\mathrm{P}<0,05)$, porém sem efeito nos valores da altura da cernelha no salto $(\mathrm{P}>0,05)$. Após o treinamento, os equinos realizaram a trajetória do salto mais próxima da ideal, demonstrando uma eficiência no treinamento realizado (Fig. 2), pois, segundo os manuais de equitação, o ponto mais alto da trajetória no salto do obstáculo do tipo oxer deve ser aproximadamente a metade da largura do obstáculo. Ao final do treinamento, observa-se que, no salto, quando a altura na cernelha era máxima, o deslocamento horizontal foi de 0,41m, próximo do valor da distância tida como ideal, de $0,45 \mathrm{~m}$, já que o obstáculo no presente estudo apresentava largura de $0,90 \mathrm{~m}$.

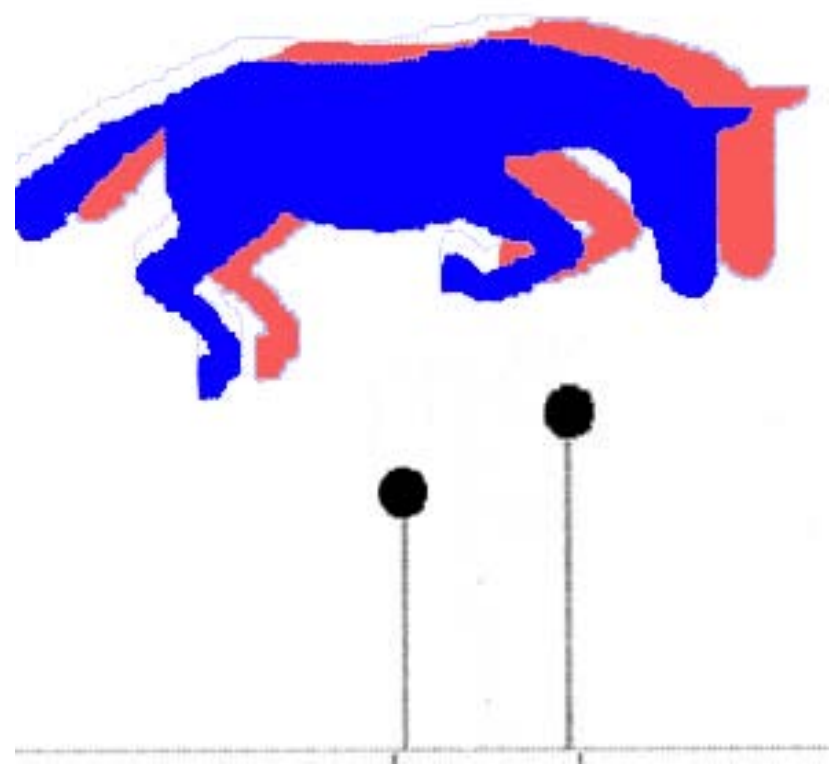

Figura 2. Posição do cavalo sobre o obstáculo antes do treinamento (vermelho) e posição do cavalo após o treinamento (azul).

Analisando os resultados, pode-se verificar que existe a possibilidade de, com treinamento específico para salto, realizar a correção de cavalos que possuem trajetória incorreta. Esse fato é de grande relevância na equitação, pois indica que a trajetória de salto do equino não é, necessariamente, uma característica inata do animal, podendo ser modificada com o treinamento.
Observa-se que o ângulo do pescoço não foi influenciado pelo treinamento (Tab. 3). Esperava-se que, com o treinamento, pudesse haver uma redução desse ângulo, ressaltando o abaixamento do pescoço em movimento de báscula para que houvesse redução dos ângulos do segmento torácico. A literatura consultada não apresenta informações sobre essa variável, que se julga de grande importância para a correta 
trajetória do salto. Existe ainda a necessidade de uma melhor definição do ponto de digitalização dessa variável, pois talvez o momento de passagem dos membros torácicos sobre o obstáculo não corresponda ao momento de maior báscula do pescoço do cavalo, sendo necessários estudos pormenorizados a respeito dessa variável. Godoi et al. (2014) observou que essa variável é importante para predizer o sucesso no salto de obstáculos por potros inexperientes.

O ângulo escapuloumeral foi a variável que apresentou maior influência do treinamento $(\mathrm{P}<0,05)$. Godoi et al. (2014), avaliando potros da raça Brasileiro de Hipismo, citam que esse ângulo é importante no salto de obstáculo, principalmente durante a fase de aterrissagem, para a absorção do impacto. E, observaram valor médio de 98,95. Santamaría et al. (2006) citam valor médio do ângulo escapuloumeral de $86,51 \pm 11,46^{\circ}$ em equinos considerados os melhores saltadores. Os valores supracitados referem-se, entretanto, a saltos realizados sobre obstáculos verticais, ao passo que, no presente trabalho, foram utilizados oxers.

Esperava-se que, com o treinamento, houvesse redução do ângulo radiometacarpiano, porém tal fato não ocorreu. Essa informação demonstra a importância que deve ser dada a esse ângulo na escolha dos equinos para a modalidade salto, uma vez que não houve modificações com o treinamento, sugerindo que seja uma característica inata do animal.

Tabela 3. Valores médios e desvio padrão das características cinemáticas angulares dos equinos no salto de obstáculo

\begin{tabular}{lccc} 
& \multicolumn{2}{c}{ Avaliação dos equinos no salto } & \\
\cline { 2 - 3 } Características cinemáticas & $\begin{array}{c}\text { Antes do } \\
\text { treinamento }\end{array}$ & $\begin{array}{c}\text { Após o } \\
\text { treinamento }\end{array}$ & Prob \\
\hline Ângulo do pescoço $\left({ }^{\circ}\right)$ & $32,4 \pm 6,4 \mathrm{a}$ & $33,2 \pm 4,7 \mathrm{a}$ & 0,5689 \\
Ângulo escapuloumeral $\left({ }^{\circ}\right)$ & $70,7 \pm 6,3 \mathrm{~b}$ & $91,4 \pm 5,8 \mathrm{a}$ & 0,0000 \\
Ângulo umerorradial $\left({ }^{\circ}\right)$ & $71,8 \pm 8,8 \mathrm{a}$ & $62,6 \pm 3,8 \mathrm{~b}$ & 0,0025 \\
Ângulo radiometacárpico $\left(^{\circ}\right)$ & $73,0 \pm 10,5 \mathrm{a}$ & $71,3 \pm 9,1 \mathrm{a}$ & 0,6306 \\
Distância escapulometacarpofalângica (m) & $0,5 \pm 0,0 \mathrm{~b}$ & $0,6 \pm 0,0 \mathrm{a}$ & 0,0168 \\
Ângulo coxofemoral $\left(\left(^{\circ}\right)\right.$ & $81,0 \pm 9,4 \mathrm{~b}$ & $90,5 \pm 5,2 \mathrm{a}$ & 0,0001 \\
Ângulo femorotibial $\left({ }^{\circ}\right)$ & $69,2 \pm 4,4 \mathrm{~b}$ & $72,0 \pm 4,0 \mathrm{a}$ & 0,0035 \\
Ângulo tibiometatársico $\left(^{\circ}\right)$ & $89,1 \pm 17,0 \mathrm{a}$ & $82,7 \pm 13,4 \mathrm{~b}$ & 0,0393 \\
Ângulo cernelhametatarsofalângica $\left(^{\circ}\right)$ & $134,5 \pm 10,9 \mathrm{a}$ & $131,3 \pm 6,1 \mathrm{a}$ & 0,2090 \\
\hline
\end{tabular}

Médias seguidas por letras diferentes nas linhas diferem pelo teste de $\mathrm{t}(\mathrm{P}<0,05)$; Prob.: probabilidade estatística.

A distância escapulometacarpofalângica deve ser a menor possível, demonstrando o fechamento ou retração máxima dos membros torácicos dos equinos sobre o salto. No presente trabalho, verificou-se que o treinamento afetou negativamente essa variável. Uma sugestão para trabalhos futuros seria a alteração do momento da avaliação da distância escapulometacarpofalângica, pois essa característica é mensurada no momento em que o boleto (articulação metacapofalângica) do membro torácico do equino está sobre a vara do segundo elemento do obstáculo oxer, momento no qual, de acordo com os resultados da variável deslocamento horizontal da cernelha no salto, os membros torácicos se preparam para o retorno ao solo (aterrissagem).

Os ângulos coxofemoral e femorotibial apresentaram influência do treinamento
$(\mathrm{P}<0,05)$. Essas variáveis estão relacionadas com a flexão dos membros pélvicos sobre o obstáculo. Estudos futuros, verificando a velocidade angular e o momento no qual esses ângulos atingem os valores máximo e mínimo, podem ser importantes para verificar a capacidade flexora dos membros pélvicos dos equinos.

Santamaría et al. (2006) verificaram que equinos com melhor aptidão para o salto apresentaram menores valores do ângulo femorotibial. É uma observação interessante, uma vez que, com a menor angulação, o animal recolhe mais os membros pélvicos, tendo menor chance de tocar no obstáculo. A redução do ângulo femorotibial com a flexão dos membros pélvicos ocorre em direção ao corpo do equino, e não para trás, como se deseja, ocasionando um salto com os membros pélvicos perto do ventre dos equinos 
(conhecido como "salto encarneirado"). Isso prejudica, principalmente, a aterissagem do cavalo e a passada do galope após o salto. No presente estudo os animais apresentaram aumento dessa angulação, demonstrando maior capacidade de estender os membros pélvicos para trás, realizando um correto gesto de salto.

O ângulo tibiometatársico apresentou influência do treinamento $(\mathrm{P}<0,05)$, demonstrando que, após o treinamento, houve redução dos valores, possibilitando aos equinos menor possibilidade de derrube do obstáculo.

O ângulo cernelhametatarsofalângica reflete a capacidade de flexionamento do dorsolombo dos equinos durante o salto. $\mathrm{O}$ treinamento ao qual os equinos foram submetidos não influenciou essa característica, diferentemente do citado por Santamaría et al. (2006), que verificaram que houve redução nos valores desse ângulo com o treinamento, possivelmente devido ao maior flexionamento dos animais.

\section{CONCLUSÃO}

Conclui-se que o treinamento específico para salto, com cinco meses de duração, altera algumas variáveis cinemáticas dos equinos no salto de obstáculo oxer, proporcionando melhoria, principalmente, na trajetória do salto.

\section{AGRADECIMENTOS}

À Escola de Equitação do Exército, ao Regimento Andrade Neves do Exército Brasileiro, à Escola de Veterinária - UFMG, à Escola de Educação Física, Fisioterapia e Terapia Ocupacional - UFMG, na pessoa do Prof. Hans-Joachim Karl Menzel, ao CNPq, à CAPES e à FAPERJ.

\section{REFERÊNCIAS}

ALLEN, L.; DENNIS, D. 101 Jumping Exercises for horse and rider. United States: INTERGLOBE, 2002. 174p.

BARREY, E. Gaits and interlimb coordination. In: BACK, W.; CLAYTON, H. (Ed). Equine Locomotion. England: ELSEVIER, 2013. p.85-98.
BOBBERT, M.; SANTAMARÍA. S.; Van WEEREN. P.R. et al. Can jumping capacity of adult show jumping horses be predicted on the basis of sub maximal free jumps at foal age? A longitudinal study. Vet. J., v.170, p.212-221, 2005.

CONFEDERAÇÃO Brasileira De Hipismo Regulamento de Salto. p.81, 2014. Disponível em: <http www.cbh.org.br >. Acessado em: 15 fev. 2014.

GODOI, F.N.; ALMEIDA, F.Q.; TORAL, F.L.B. et $a l$. Repeatability of kinematics traits of free jumping in Brazilian Sport horse. Lives. Sci., v.168, p.01-08, 2014.

GODOI, F.N.; BERGMANN, J.A.G.; ALMEIDA, F.Q. et al. Morfologia de potros da raça Brasileiro de Hipismo. Cienc. Rur., v.43, p.736-742, 2013.

KOENEN, E.P.C.; ALDRIDGEB, L.I.; PHILIPSSON, J. An overview of breeding objectives for warmblood sport horses. Liv. Prod. Sci., v.88, p.77-84, 2004.

LEWCZUK, D. Young horse response on changing distance in free jumping combination. Anim., v.2, p.1651-1657, 2008.

LEWCZUK, D.; DUCRO, B. Repeatability of free jumping parameters on tests of different duration. Liv. Sci., v.146, p.22-28, 2012.

LEWCZUK, D.; SLONIEWSKI, Z.; REKLEWSKI, Z. Repeatability of the horse's jumping parameters with and without the rider. Liv. Sci., v.99, p.125-130, 2006.

POWERS, P.; HARRISON, A. A study on the techniques used by untrained horses during loose jumping. J. Eq.Vet. Sci., v.20, p.845-850, 2000.

SANTAMARÍA, S.; BOBBERT, M.F.; BACK, W. et al. Effect of early training on the jumping technique of horses. Am. J. Vet. Res., v.66, p.418-424, 2005.

SANTAMARÍA, S.; BOBBERT, M.E.; BACK, W. et al. Evaluation of consistency of jumping technique in horses between the ages of 6 months and 4 years. Am. J. Vet. Res., v.65, p.945-950, 2004.

SANTAMARÍA, S.; BOBBERT, M.F.; BACK, W. et al. Can early training of show jumpers bias outcome of selection events? Liv. Sci., v.102, p.163-170, 2006.

SCHALLER, O. Nomenclatura anatômica veterinária ilustrada. Brasil: MANOLE, 1999. 560p.

WEJER, J.; LENDO, I.; LEWCZUK, D. The effect of training on the jumping parameters of inexperienced Warmblood horses in free jumping. J. Eq. Vet. Sci., v.33, p.483-486, 2013. 